\title{
BMJ Open Efficacy and acceptability of adjunctive psychological and pharmacological interventions for treatment-resistant depression: protocol for a systematic review and network meta-analysis
}

Muhammad Ishrat Husain, ${ }^{1}$ Rebecca Strawbridge, ${ }^{2}$ Ben Carter, ${ }^{3}$ Brett D M Jones, ${ }^{1}$ Allan Young, ${ }^{2}$ Andre F Carvalho ${ }^{1}$

To cite: Husain MI,

Strawbridge R, Carter B, et al. Efficacy and acceptability of adjunctive psychological and pharmacological interventions for treatment-resistant depression: protocol for a systematic review and network meta-analysis. BMJ Open 2019;9:e028538. doi:10.1136/ bmjopen-2018-028538

- Prepublication history for this paper is available online. To view these files please visit the journal online (http://dx.doi. org/10.1136/bmjopen-2018028538).

Received 12 December 2018 Revised 17 April 2019 Accepted 17 April 2019

Check for updates

(C) Author(s) (or their employer(s)) 2019. Re-use permitted under CC BY-NC. No commercial re-use. See rights and permissions. Published by BMJ.

${ }^{1}$ General Psychiatry and Health Systems Division, Centre for Addiction and Mental Health, Toronto, Ontario, Canada ${ }^{2}$ Department of Psychological Medicine, King's College London, London, UK

${ }^{3}$ Biostatistics \& Health Informatics, King's College London, London, UK

Correspondence to Dr Muhammad Ishrat Husain; Ishrat.husain@camh.ca

\section{ABSTRACT}

Introduction Major depressive disorder (MDD) is a common debilitating illness worldwide. The vast majority of patients with MDD will not achieve remission with first-line treatment and despite the availability of different treatment modalities, at least one-third of patients experience treatment-resistant depression (TRD). There continues to be a paucity of research focused on treatment options for patients with TRD thus treatment decisions are largely based on patient and clinician preference as opposed to evidence-based practice. Herein we propose a systematic review and network meta-analysis (NMA) of available pharmacological and psychological augmentation treatments for TRD, to inform evidencebased management of TRD.

Methods and analysis We plan to conduct a search of electronic databases (MEDLINE and ISIWEB) of all dates from inception for randomised controlled trials of pharmacological and psychological augmentation interventions for adults with TRD. Articles for review will be included based upon consensus from two authors. Pharmaceutical companies will be contacted for access to any unpublished data. An NMA will compare the effectiveness pharmacological adjunctive agents for TRD using preanalysis/postanalysis, assuming consistency and transitivity.

Ethics and dissemination This project does not require research ethics board approval. The dissemination plan is to present findings at international scientific meetings and publishing results in a peer-reviewed academic journal. PROSPERO registration number CRD42019132588.

\section{INTRODUCTION}

As of 2017, the WHO classifies major depressive disorder (MDD) as the leading cause of disability worldwide. ${ }^{1}$ Economic estimates report that the annual attributable financial loss due to MDD is US $\$ 83$ billion. ${ }^{2}$ Though there are effective treatments for MDD, those who seek treatment are often faced with a relapsing and recurring course of illness. Based on community surveys, the finding
Strengths and limitations of this study

- This will be the most comprehensive review of published and unpublished data of pharmacological and psychological augmentation treatments for treatment-resistant depression (TRD).

- The results will provide the highest level of evidence to inform clinicians on the best choice of treatment from among the available pharmacological and psychological interventions for TRD.

- The reporting of the protocol has been guided by Preferred Reporting Items for Systematic Reviews and Meta-Analyses and has been registered with International Prospective Register of Systematic Reviews.

- The study does not include brain stimulation interventions and trials of agents used as monotherapy.

that lifetime prevalence is two to three times that of 12-month prevalence suggests that between one-third and one-half of lifetime cases have recurrent episodes in a given year. ${ }^{3}$ The STAR*D study, which was the largest naturalistic study on treatments for MDD to date, indicated that remission rates on the first treatment trial were approximately one-third and subsequent remission rates decreased as the number of treatment trials increased. ${ }^{4}$ The Sequenced Treatment Alternatives to Relieve Depression (STAR*D) findings indicate that at least a third of patients are likely to be experiencing treatment-resistant depression (TRD). Those suffering from TRD are left suffering from a significant decline in their social and occupational functioning and higher rates of all-cause mortality. ${ }^{5}$ Persistent symptoms in TRD often translate into exponential increases in work loss and medical costs compared with more responsive forms of illness. 
In the clinical setting, very few patients are treatment naive or are experiencing their first major depressive episode, yet the vast majority of research on treatment for MDD has focused on single-episode depression. Very few studies have looked at the specific patient population that is treatment resistant. When a patient presents as refractory to first-line antidepressant (AD) medication, a crucial clinical question is whether to augment, make an $\mathrm{AD}$ switch or switch treatment modalities. A recent review outlined the current evidence-base and treatment modalities available for TRD. ${ }^{6}$ Despite the summary of evidence, it does not provide guidance as to whether individuals should receive augmentation, discontinuation or switch to alternative treatment strategies. This decision remained largely dependent on patient and clinician preference. ${ }^{6}$ Although there have been recent network meta-analyses published attempting to answer this clinical question, they have been limited by either setting a loose definition of TRD (ie, one failed treatment only), restricting the search to a narrow range of publication dates, excluding unpublished data, excluding trials of psychological interventions and excluding trials of novel treatment options such as anti-inflammatory agents. ${ }^{7-9}$ Therefore, we aim to address these limitations and herein present the protocol for a network meta-analysis (NMA) of current available evidence of both psychological and pharmacological augmentation treatments for TRD.

\section{OBJECTIVE}

To assess and compare the effectiveness of psychological and pharmacological augmentation treatments for TRD using a NMA approach. Since NMA combines evidence based on both direct and indirect comparison, it maximises data included in analyses and provides relative estimates of effectiveness of all interventions considered. Specifically we aim to:

1 . Determine the effectiveness of all psychological and pharmacological adjunctive agents for TRD, in comparison to one another.

2. Determine the acceptability and tolerability of these treatments.

\section{METHODS AND ANALYSIS}

The reporting of the protocol has been guided by the Preferred Reporting Items for Systematic Reviews and Meta-Analyses statement. ${ }^{10}$

\section{Patient and public involvement}

Treatment resistant depression is a major public health concern and contributes to persistent suffering and frustration at an individual patient level. Our findings will help address this concern to better serve the patients we see in practice. Since this is a secondary analysis, it was felt at this time, patient and public involvement was not required to inform the design of this particular study. As described above, we will widely disseminate our findings to reach the highest impact.

\section{Eligibility criteria}

Participants and setting

We will include studies that recruited:

- Participants of any gender.

- Participants aged $>18$. It may be that treatment efficacies differ for patients with late-life depression; as such, studies recruiting patients solely over age 65 will be excluded. Furthermore, we will conduct a sensitivity analysis to determine the effect of age on outcomes.

- We will exclude studies that enrolled patients with comorbid neurological disorders.

- Any setting can be included and these data are extracted for potential network meta-regression.

- Studies must have required participants to have TRD, defined as meeting all of the following criteria:

a. Currently meets diagnostic criteria for MDD according to ICD 10 Code F32-3, DSM-IV 296 and DSM-5 296. ${ }^{11-13}$ Trials with ICD 9and DSM III/IIIR diagnoses approximating to these codes are also considered appropriate.

b. Within the current depressive episode, have not sufficiently responded to at least two adequate trials of an evidenced based treatment for depression, as used in the most popular TRD definitions. ${ }^{14-16}$ Any studies that include non-response to within-class AD switches (eg, two SSRI's, or two SNRI's) as contributing to TRD will be included, due to evidence demonstrating that failure to respond after within-class $\mathrm{AD}$ switch should contribute to the staging of TRD. ${ }^{17}$ Similarly, we consider failure to respond to an adequate course of a psychological treatment to also contribute to TRD staging and thus this will be eligible for inclusion. ${ }^{18}$ For instance, if patients have not responded to adequate trials of a Selective Serotonin Reuptake Inhibitor (SSRI) and cognitive behavioural therapies (CBT), this will be eligible for inclusion in the present study. In line with commonly used TRD definitions, we will only consider treatment resistance within a current episode.

We will exclude:

- Studies of children or adolescents $(<18$ years of age), due to differing treatment mechanisms in young people, particularly with regard to ADs.

- Studies where $\mathrm{n}<10$ participants randomised. ${ }^{19}$

- Studies including patients with bipolar disorder and psychoses will be excluded (unless data are available for the subgroup of non-psychotic, unipolar participants), due to the well-documented differences in treatment efficacy for patients with these syndromes. However, multiple comorbidities are extremely common in TRD and therefore we will permit the inclusion of studies not excluding participants with most psychiatric and physical comorbidities. 


\section{Interventions}

Permitted pharmacological treatments include those included in the Maudsley Treatment Inventory (MTI) derived from the Maudsley Prescribing Guidelines and other standardised guidelines for depression treatment. We also include any treatment that has multiple meta-analyses supporting its use (eg, minocycline, which is not included in the MTI). ${ }^{20}$

For psychological agents, there has been far less standardisation and definition of appropriate AD therapies. Permitted psychological treatments will comprise those recommended in the most recent National Institute of Clinical Excellence (NICE) guidelines for depression (those only recommended for mild depression are not included) and comprise: computerised or face-to-face CBT, behavioural activation, interpersonal therapy, manualised psychodynamic therapy, behavioural couples therapy, cognitive behavioural analysis system of psychotherapy, or mindfulness-based cognitive therapy.

Due to the variation between treatments in duration required for clinical effectiveness, trials will be considered adequate and therefore included if the article reports expectations of clinical efficacy.

These are the criteria for both augmentation and initial treatments. While any evidence-based initial treatment is permitted (pharmacological or psychological) it is anticipated that the majority of, if not all, studies will have recruited medicated TRD patients.

\section{Comparators}

Eligible comparator treatments include: Placebo, another pharmacological agent, another psychological intervention, waiting list, active control or treatment-as-usual.

\section{Outcomes}

We will only include studies that report depression-rating scales in each treatment arm.

\section{Study designs and publication types}

Randomised controlled trials (RCTs), cluster RCTs or the first phase of randomised cross-over trials. Trials must include randomisation to at least one suitable augmentation treatment (where patients are taking at least one continuing treatment prior to randomisation to further intervention). Eligibility of both initial and augmentation treatments is described below.

\section{Language and time frame}

For any articles not available in English, maximum efforts will be made to translate text such that all studies can be included. The search will be created from all years up to the date of systematic search, 21 December 2018. We intend for the analysis and drafting of the manuscript to be reviewed and submitted to publication by 1 September 2019.

\section{Information source and search strategy}

Electronic databases MEDLINE, www.clinicaltrials.gov, and ISI Web of Science will be searched along with citation lists from notable papers, available reviews and subsequently of included articles. The following search terms are applied:

(depress* OR MDD OR major depress*) AND

(resistan* OR refractor* OR non-respon* OR nonrespon* OR un-respon* OR unrespon* OR TRD OR fail* OR inadequate OR difficult OR intractable) AND

(augment* OR adjunct* OR add-on OR combin* OR co-administ*) AND

(randomi* OR RCT) AND

(treatment OR intervention OR trial).

For the NMAs, the systematic search will be maximised by contacting pharmaceutical companies and additional authors in an attempt to collate data from unpublished studies.

\section{Study selection}

All studies generated from the systematic searches will be evaluated against the predefined inclusion criteria by two of the review authors independently. Any disparities will be addressed by reaching agreement via two additional review authors. We will use the GRADE approach to rank the quality of available evidence.

\section{Data extraction}

This will be conducted by two review authors independently and will include quality assessment, description of participants, description of the intervention and control groups, psychometric data and outcomes. Disagreements will be resolved via further discussion with a third review author.

\section{Assessment of risk of bias}

The methodological quality and specifically risk of bias, of included studies, will be addressed by employing the Scottish Intercollegiate Guidelines Network tool in conjunction with the Cochrane risk of bias tool. Two authors will extract these data independently, as above. The data extracted will relate to quality across nine domains: sequence generation, allocation concealment, blinding of outcome assessors, use of intention-to-treat (ITT) analysis, comparability of randomised groups at baseline, inter-site differences in findings, the potential for selective outcome reporting and presence of for-profit bias (allegiance). Additional sources of bias that will be assessed will include novelty bias and sponsorship bias. A sensitivity analysis will assess the difference in statistical effects between studies with a high and low risk of bias.

\section{Outcome measure(s)}

Primary outcome(s)

The primary outcome will be treatment effectiveness using any validated assessment of illness severity, which is repeated from baseline to endpoint. Where multiple outcomes are reported, the primary outcome for inclusion in analyses will be selected in a hierarchical fashion: the most preferable scale will be a clinician-rated assessment of depression severity (Hamilton Rating Scale for Depression [HAM-D], Montgomery-Asberg Depression 
Rating Scale [MADRS], Inventory of Depressive Symptomatology [IDS] or validated subscales of these), followed by a patient-rated measure (Patient Health Questionnaire [PHQ-9], IDS or Beck Depression Inventory [BDI]) or if not available, an assessment of global improvement (such as the Clinical Global Impression [CGI] scale, LIFE chart measure or Global Assessment of Functioning [GAF]) or related symptoms (such as anxiety). Where multiple endpoints are reported, this review will consider the primary endpoint reported by each study.

\section{Secondary outcome(s)}

1 . Response rate measured by the total number of patients who had a reduction of $\geq 50 \%$ of the total score on a standardised rating scale for depression.

2. Remission rates as measured by a standardised rating scale for depression.

3. All-cause discontinuation will be used as a measure for the acceptability of treatments, because it encompasses efficacy and tolerability.

\section{Statistical analysis}

Aggregate data (as opposed to individual patient level) will likely need to be used for the present quantitative analyses. Descriptive statistics of study and participant characteristics will be examined and studies must be sufficiently homogeneous to be included in analyses. Pooled effect sizes (ES) will be calculated from continuous (standardised mean differences; Hedges' g. SD will be input where possible, or imputed from SE, CI $(95 \% \mathrm{CI})$ or $p$ values.

We will generate a network plot, consisting of all of the direct evidence, weighted by number of number of studies connecting the nodes. Relative effectiveness will be examined by calculating relative ES (probability of each treatment to be best). These probabilities are expressed by a Surface under the Cumulative Ranking curve percentage, used to create the probability of the best treatment option, across all interventions.

Depending on data availability, we may attempt to explore potential explanatory factors using sensitivity analysis/metaregression. Examples of potentially important variables include: study quality, extent of treatment resistance, initial treatments, comorbidities, age, gender or ethnicity of sample, depression severity, duration of episode, duration of intervention, treatment setting. Novelty bias, sponsorship bias, definition or criteria used for TRD. These should be included as possible sources of inconsistency.

\section{Assumptions of the NMA}

The comparator groups will be explored for homogeneity of clinical context. Where these are considered to not exhibit contextual diversity, the assumptions of transitivity and similarity will be compared by observation between direct and indirect comparisons. An ITT approach will be taken to missing data. Heterogeneity of the network will be measured using $\mathrm{I}^{2}$, and $\tau^{2}$ within and across comparisons.

\section{Limitations}

One of the main limitations of this review is that it only includes only of trials of augmentation treatments, thus excluding many of the ketamine investigations which have been trialled as a monotherapy. We also did not include trials of brain stimulation interventions, which are sometimes used with good effect for TRD. Since brain stimulation interventions are excluded, the findings may over-represent patients who are inappropriate for, or lack access to, brain stimulation treatments. Our search strategy was limited to MEDLINE, Clinicaltrials.gov and ISI Web of Science although we expect adequate and efficient coverage with these databases. While interpreting the meta-analysis, we have to consider the heterogeneity of the dose/intensity and duration of the intervention, which is not always standardised across interventions. Network meta-analyses make both direct and indirect comparisons and we will aim to assess for inconsistencies of these two comparisons by using the node-splitting method in our statistical analysis.

\section{Ethics and dissemination}

This project does not require research ethics board approval as it does not directly involve human or animal participants and is a review of available literature. We plan to present our findings at national and international scientific meetings. We also plan to publish our results in a reputable peer-reviewed academic journal.

Contributors MIH contributed to the methodology and led the first draft of the protocol. RS developed the initial methodology and helped write the first draft of the protocol. BDMJ contributed to the first draft of the manuscript, is updating searches and is obtaining unpublished data from pharmaceutical companies. BC contributed to the methodology and statistical analysis plan. AY and AFC conceived the idea for this network meta-analysis and provided critical feedback on the search strategy, methodology and manuscript. All authors approved the final version of the manuscript.

Funding This study represents independent research partly funded by the National Institute for Health Research (NIHR) Biomedical Research Centre at South London and Maudsley NHS Foundation Trust and King's College London.

Competing interests AY has received honoraria for speaking from Astra Zeneca, Lundbeck, Eli Lilly, Sunovion; honoraria for consulting from Allergan, Livanova and Lundbeck, Sunovion, Janssen; and research grant support from Janssen, in the last 3 years.

Patient consent for publication Not required.

Provenance and peer review Not commissioned; externally peer reviewed.

Open access This is an open access article distributed in accordance with the Creative Commons Attribution Non Commercial (CC BY-NC 4.0) license, which permits others to distribute, remix, adapt, build upon this work non-commercially, and license their derivative works on different terms, provided the original work is properly cited, appropriate credit is given, any changes made indicated, and the use is non-commercial. See: http://creativecommons.org/licenses/by-nc/4.0/.

\section{REFERENCES}

1. WHO. Depression and other common mental disorders global health estimates, 2017. 
2. Greenberg PE, Kessler RC, Birnbaum HG, et al. The economic burden of depression in the United States: how did it change between 1990 and 2000? J Clin Psychiatry 2003;64:1465-75.

3. Kessler RC, Bromet EJ. The epidemiology of depression across cultures. Annu Rev Public Health 2013;34:119-38.

4. Rush JA, Jain S. Clinical Implications of the STAR*D Trial. Berlin, Heidelberg: Springer, 2018:1-49.

5. Reutfors J, Andersson TM, Brenner P, et al. Mortality in treatmentresistant unipolar depression: A register-based cohort study in Sweden. J Affect Disord 2018;238:674-9.

6. Mclntyre RS, Filteau MJ, Martin L, et al. Treatment-resistant depression: definitions, review of the evidence, and algorithmic approach. J Affect Disord 2014;156:1-7.

7. Papadimitropoulou K, Vossen C, Karabis A, et al. Comparative efficacy and tolerability of pharmacological and somatic interventions in adult patients with treatment-resistant depression: a systematic review and network meta-analysis. Curr Med Res Opin 2017;33:701-11.

8. Zhou X, Keitner GI, Qin B, et al. Atypical Antipsychotic Augmentation for Treatment-Resistant Depression: A Systematic Review and Network Meta-Analysis. Int J Neuropsychopharmacol 2015;18:pyv060.

9. Zhou X, Ravindran AV, Qin B, et al. Comparative efficacy, acceptability, and tolerability of augmentation agents in treatmentresistant depression: systematic review and network meta-analysis. $J$ Clin Psychiatry 2015;76:e487-98.

10. Shamseer L, Moher D, Clarke M, et al. Preferred reporting items for systematic review and meta-analysis protocols (PRISMA-P) 2015: elaboration and explanation. BMJ 2015;349:g7647.

11. WHO. The ICD-10 Classification of Mental and Behavioural Disorders Clinical descriptions and diagnostic guidelines: World Health Organization, 1992.
12. First M, Gibbon M, Spitzer R, et al. User Guide for the Structural Clinical Interview for DSM-IV Axis I Disorders. APA. Washington, DC: American Psychiatric Association, 1996.

13. American Psychiatric Association. Diagnostic and statistical manual of mental disorders. 5th ed. Washington, DC: Author, 2013.

14. Anderson IM, Ferrier IN, Baldwin RC, et al. Evidence-based guidelines for treating depressive disorders with antidepressants: a revision of the 2000 British Association for Psychopharmacology guidelines. J Psychopharmacol 2008;22:343-96.

15. NICE. Depression in Adults (update) Depression: the treatment and management of depression in adults National Clinical Practice Guideline Number X National Collaborating Centre for Mental Health Commissioned by the National Institute for Health and Clinical Exc, 2007.

16. Souery D, Amsterdam J, de Montigny C, et al. Treatment resistant depression: methodological overview and operational criteria. Eur Neuropsychopharmacol 1999;9:83-91.

17. Papakostas GI, Fava M, Thase ME. Treatment of SSRI-resistant depression: a meta-analysis comparing within- versus across-class switches. Biol Psychiatry 2008;63:699-704.

18. Wiles N, Thomas L, Abel A, et al. Cognitive behavioural therapy as an adjunct to pharmacotherapy for primary care based patients with treatment resistant depression: results of the CoBalT randomised controlled trial. Lancet 2013;381:375-84.

19. Bandelow B, Reitt M, Röver C, et al. Efficacy of treatments for anxiety disorders. Int Clin Psychopharmacol 2015;30:183-92.

20. Fekadu A, Donocik JG, Cleare AJ. Standardisation framework for the Maudsley staging method for treatment resistance in depression. BMC Psychiatry 2018;18:100. 